\title{
Invadopodia are essential in transurothelial invasion during the muscle invasion of bladder cancer cells
}

\author{
KENGO IMANISHI $^{1}$, MIHOKO SUTOH YONEYAMA ${ }^{1,2}$, SHINGO HATAKEYAMA $^{1}$, HAYATO YAMAMOTO ${ }^{1}$, \\ TAKUYA KOIE $^{1}$, HISAO SAITOH ${ }^{3}$, KANEMITSU YAMAYA ${ }^{3}$, TOMIHISA FUNYU ${ }^{3}$, \\ TOSHIYA NAKAMURA ${ }^{4}$, CHIKARA OHYAMA ${ }^{1}$ and SHIGERU TSUBOI ${ }^{1,2}$ \\ ${ }^{1}$ Department of Urology, Hirosaki University Graduate School of Medicine, Hirosaki, Aomori 036-8562; \\ Departments of ${ }^{2}$ Cancer Immunology and Cell Biology and ${ }^{3}$ Urology, Oyokyo Kidney Research Institute, Hirosaki, \\ Aomori 036-8243; ${ }^{4}$ Department of Biomedical Sciences, Hirosaki University Graduate School of Health Sciences, \\ Hirosaki, Aomori 036-8564, Japan
}

Received August 31,2013; Accepted March 6, 2014

DOI: $10.3892 / \mathrm{mmr} .2014 .2113$

\begin{abstract}
Muscle invasive bladder cancer is an aggressive type of epithelial tumor with a high rate of metastasis. For bladder cancer cells to reach the muscle layer, cells must invade through an urothelial cell monolayer (transurothelial invasion) and basement membrane. However, the process by which transurothelial invasion occurs has not been fully characterized. In this study we developed a novel method to evaluate the transurothelial invasion capacity and investigated its cellular and molecular processes using primary culture cells from bladder cancer patients. The analysis revealed that compared with the prognosis for patients with non-muscle invasive bladder cancer that of patients with muscle invasive bladder cancer was particularly poor due to metastatic recurrence. Cancer cells from patients with muscle invasive bladder cancer exhibited a higher invasive capacity through the urothelial cell monolayer compared with those from non-invasive bladder cancer patients. Furthermore, muscle invasive bladder cancer cells demonstrated a greater ability to form invadopodia, the filamentous actin-based membrane protrusions required for matrix degradation and invasion compared with non-invasive cells. Bladder cancer cell lines were established with reduced invadopodia formation by silencing the expression of cortactin, an essential component of invadopodia. The cortactin knockdown bladder cancer cells with reduced invadopodia formation demonstrated a markedly reduced ability to invade through the urothelial cell monolayer, indicating that invadopodia are essential for transurothelial invasion. The results indicate that invadopodia formation is required for muscle invasion of aggressive bladder cancer cells.
\end{abstract}

Correspondence to: Dr Shigeru Tsuboi, Department of Urology, Hirosaki University School of Medicine, 5 Zaifu-cho, Hirosaki, Aomori 036-8562, Japan

E-mail: urology@cc.hirosaki-u.ac.jp

Key words: bladder cancer, metastasis, transurothelial invasion, muscle invasive, invadopodia

\section{Introduction}

The majority of cancer fatalities are due to metastatic recurrence. Hematogenous metastasis of aggressive bladder cancer involves the following processes. i) Invasive bladder cancer cells escape from the primary site, the bladder, to invade a muscle layer (muscle invasion). ii) The muscle-invaded cancer cells enter nearby blood vessels by degrading the basement membrane and transmigrating through an endothelial cell monolayer (intravasation). iii) Cells disseminate around the body using the circulatory system of the host. iv) The circulating cancer cells exit the vessels to invade, via the endothelial cell monolayer, the tissue of a secondary organ (extravasation) and subsequently proliferate to form metastasis (1). Of the numerous factors involved in the multiple processes, it was previously demonstrated that cancer cells expressing an $O$-glycan branching enzyme (core2 $\beta-1,6-N$ acetylglucosaminyltransferase) evade natural killer (NK) cell immunity to acquire highly metastatic phenotypes by surviving longer in the circulatory system of the host (2-4).

Bladder cancers are heterogeneous entities comprised of non-muscle invasive and muscle invasive bladder cancers. Muscle invasive bladder cancer is an aggressive type of epithelial tumor with a high rate of early systemic dissemination and poor patient prognosis (5). An improved understanding of the process of muscle invasion of bladder cancer is highly desirable. In order for aggressive bladder cancer cells to invade the muscle layer from the bladder epithelia, the cancer cells must invade through a urothelial cell monolayer (transurothelial invasion) and the basement membrane. In the present study, a novel method was developed to evaluate the invasion of the bladder cancer cells through the urothelial cell monolayer, a crucial process for muscle invasion, and investigated the cellular mechanisms underlying transurothelial invasion.

\section{Materials and methods}

Clinical data analysis. Between January 1996 and October 2012 radical cystectomies were performed on 324 consecutive 
patients with bladder cancer at the Department of Urology, Hirosaki University Graduate School of Medicine (Hirosaki, Japan). Histopathological grading was performed according to the World Health Organization system (6). Bladder tumor specimens were fixed with $10 \%$ buffered formalin for $12 \mathrm{~h}$. The paraffin-embedded samples $(3 \mu \mathrm{m})$ were subjected to hematoxylin and eosin staining. Written consent was obtained from all patients and the study was approved by the institutional ethics committees of Hirosaki University. Patients were divided into non-muscle invasive or muscle invasive groups according to the pathological state of invasion. The overall survival was estimated by the Kaplan-Meier method and compared between the two groups using the log-rank test.

Primary culture of bladder cancer cells. Bladder tumors were surgically removed from certain genetically independent patients at the Department of Urology, Hirosaki University Graduate School of Medicine. Tumors were incubated with RPMI-1640 medium (Sigma-Aldrich, St. Louis, MO, USA) containing 5\% fetal bovine serum (FBS; PAA Laboratories, Morningside, QLD, Australia) and $0.1 \%$ collagenase at $37^{\circ} \mathrm{C}$ for $16 \mathrm{~h}$ to prepare single-cell suspensions. The primary culture cells were subjected to various assays and immunostaining.

Cells, reagents and antibodies. A human invasive and high-grade bladder cancer cell line, YTS-1, was provided by Dr H Kakizaki (Yamagata University, Yamagata, Japan). The RT4 cell line was purchased from the American Type Culture Collection (ATCC, Manassas, VA, USA). YTS-1 and RT4 cells were maintained in RPMI-1640 medium supplemented with $10 \%$ FBS with $5 \% \mathrm{CO}_{2}$ at $37^{\circ} \mathrm{C}$. Primary human urothelial cells (HUCs) were purchased from ScienCell Research Laboratories (Carlsbad, CA, USA) and maintained in urothelial cell media-basal (UCM-b; ScienCell Research Laboratories). All of the biochemical reagents were purchased from Sigma-Aldrich, unless otherwise stated. Anti-cortactin monoclonal antibody (clone EP1922Y) and anti-actin polyclonal antibody were purchased from Epitomics Inc. (Burlingame, CA, USA) and Sigma-Aldrich, respectively.

Stable transfectants. YTS-1 cells with reduced cortactin expression were generated by shRNA technology as described previously (2). An shRNA expression plasmid was constructed using pBAsi-hU6Neo DNA (Takara Bio Inc., Shiga, Japan). The shRNA sequence for cortactin was as follows: GATCCGCACGAGTCACAGAGAGATCTGTG AAGCCACAGATGGGATCTCTCTGTGACTCGTGCTT TTTTA (the siRNA sequence for cortactin is underlined). A human non-targeting siRNA sequence (Accell siRNA control; Thermo Scientific, Rockford, IL, USA) was used to prepare the control cells expressing non-targeting shRNA. The shRNA expression plasmids, knockdown and control constructs, together with pTK-HyB (Takara Bio Inc.) were introduced to the YTS-1 cells in a 10:1 molar ratio using Lipofectamine 2000 (Invitrogen Life Technologies, Carlsbad, CA, USA). Drug-resistant colonies were selected in the presence of $200 \mu \mathrm{g} / \mathrm{ml}$ hygromycin B (Sigma-Aldrich). Two knockdown clones (designated cortKD-1 and -2) were selected based on their cortactin expression levels. cortKD-1, -2 and a control clone (designated YTS control) were used for the assays described in the present study.

Western blotting. Total cancer cell lysates were prepared by solubilization in $50 \mathrm{mM}$ Tris- $\mathrm{HCl}$ buffer, $\mathrm{pH} 7.5$, containing $1 \%$ IgepalCA-630, $150 \mathrm{mM} \mathrm{NaCl}$ and proteinase inhibitors $(10 \mu \mathrm{g} / \mathrm{ml})$. The lysates were resolved by SDS-PAGE on an $8-16 \%$ gradient gel (Invitrogen Life Technologies), and transferred to a polyvinylidene fluoride membrane. Western blot analysis was performed using the specific primary antibodies (anti-cortactin antibody and anti-actin antibody) and a horseradish peroxidase-conjugated secondary antibody (GE Healthcare, Little Chalfont, UK). Signals were visualized using the ECL Plus detection system (GE Healthcare, Little Chalfont, UK).

Immunofluorescence microscopy. Cells seeded on coverslips were fixed in $4 \%$ paraformaldehyde and permeabilized with phosphate-buffered saline (PBS) containing $0.1 \%$ saponin and $1 \%$ bovine serum albumin. Cells were stained with Alexa Fluor ${ }^{\circledR}$ 568-labeled phalloidin (Invitrogen Life Technologies) together with the monoclonal antibody to cortactin, and Alexa Fluor ${ }^{\circledR}$ 488-labeled secondary antibody (Invitrogen Life Technologies) was used for antibody staining. Cell staining was examined under an Olympus IX-71 fluorescence microscope (Olympus Inc., Tokyo, Japan) and LSM 710 laser scanning confocal microscope (Carl Zeiss, Oberkochen, Germany).

Gelatin zymography. Gelatin zymography was performed in $10 \%$ Novex $^{\circledR}$ Zymogram precast SDS-PAGE gel (Invitrogen Life Technologies) in the presence of $0.1 \%$ gelatin under non-reduced conditions. Cells $\left(1 \times 10^{6}\right)$ in $2 \mathrm{ml}$ RPMI-1640 containing $10 \%$ FBS were placed in a single well (6-well dish) and grown to $80 \%$ confluence. Cells were washed with PBS and subsequently incubated with $2 \mathrm{ml} \mathrm{Opti-MM^{ \circledR }}$ (Invitrogen Life Technologies) for $24 \mathrm{~h}$. Conditioned media were collected and subjected to SDS-PAGE. Gels were washed in $2.5 \%$ Triton $\mathrm{X}-100$ for $30 \mathrm{~min}$ at room temperature in order to remove SDS, incubated at $37^{\circ} \mathrm{C}$ overnight in a substrate buffer containing $50 \mathrm{mM}$ Tris- $\mathrm{HCl}, \mathrm{pH} 8.0$, $5 \mathrm{mM} \mathrm{CaCl}_{2}$ and subsequently stained with $0.5 \%$ Coomassie Brilliant Blue R-250 in 50\% methanol and 10\% acetic acid for $1 \mathrm{~h}$.

Matrigel invasion assay. The Matrigel matrix gel invasion assay was performed using a Transwell system (BD Biosciences, San Jose, CA, USA). The bottom surface of the filter $(8-\mu \mathrm{m}$ pore size) of the upper chamber was coated with $100 \mu \mathrm{g} / \mathrm{ml}$ fibronectin and the top surface was covered with $1 \mathrm{mg} / \mathrm{ml}$ Matrigel matrix. The lower chamber was filled with serum-free RPMI-1640 medium. Bladder cancer cells $\left(5 \times 10^{4}\right)$ were labeled using a Vybrant ${ }^{\circledR}$ carboxyfluorescein diacetate succinimidyl ester (CFDA SE) Cell Tracer kit (Invitrogen Life Technologies) and subsequently placed into the upper chamber. Following incubation at $37^{\circ} \mathrm{C}$ for $24 \mathrm{~h}$, non-migrated cells that remained on the top surface of the filter were carefully removed with cotton swabs. Migrated cells on the bottom surface were fixed with 


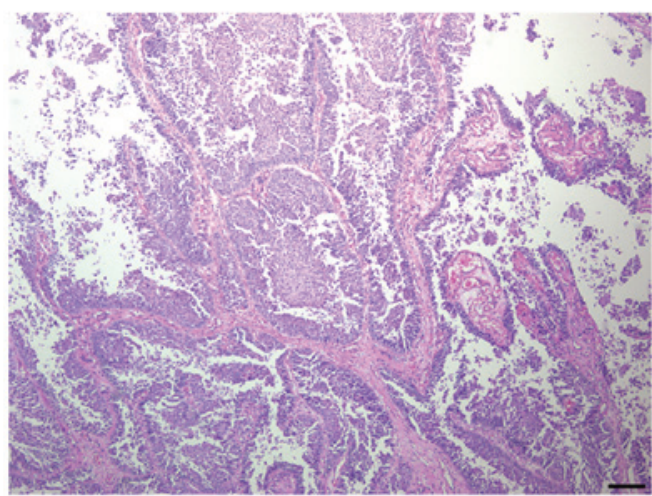

$\mathbf{C}$

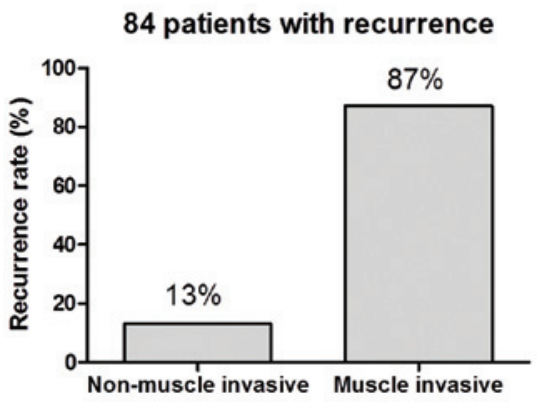

B

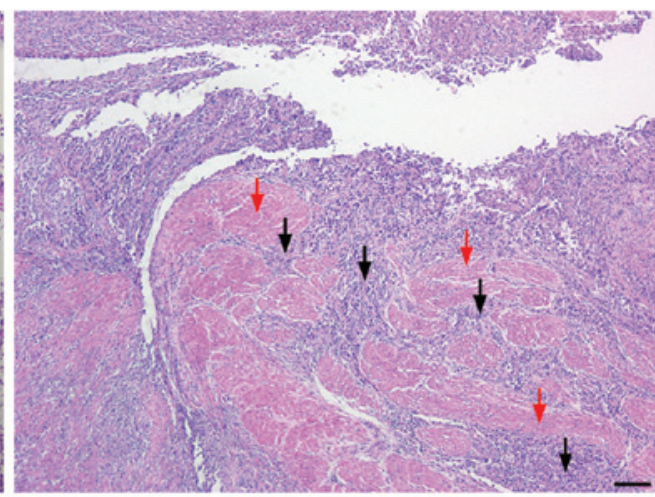

D

Overall Survival

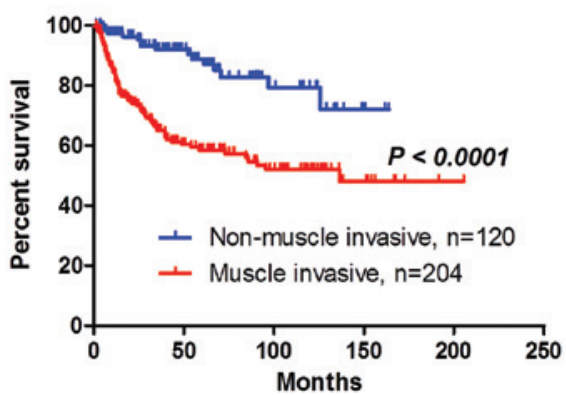

Figure 1. Poor prognosis for patients with muscle invasive bladder cancer. (A) Histology of non-muscle invasive bladder cancer. (B) Histology of muscle invasive bladder cancer. Red arrows indicate the muscle tissues and black arrows indicate the typical muscle invasive bladder cancer tissues. Scale bars, $100 \mu \mathrm{m}$. (C) Recurrence rates of bladder cancer in the non-muscle invasive cancer and muscle invasive cancer groups. (D) Kaplan-Meier curve for the overall survival of bladder cancer patients according to the degree of muscle invasiveness. Patients $(n=324)$ were divided into two groups, non-muscle invasion group ( $\mathrm{n}=120)$ and muscle invasion group $(\mathrm{n}=204)$.

4\% paraformaldehyde and counted under a Olympus IX-71 fluorescence microscope.

Transurothelial invasion assay. The transurothelial invasion assay was performed using the Transwell system. The upper surface of the filter ( $8-\mu \mathrm{m}$ pore size) of the upper chamber was coated with $100 \mu \mathrm{g} / \mathrm{ml}$ collagen I. HUCs $\left(1 \times 10^{5}\right)$ were placed onto the upper chamber and cultured for 2 days in order to form a monolayer. CFDA SE-labeled bladder cancer cells $\left(5 \times 10^{4}\right)$ were re-suspended in UCM-b medium and placed onto the HUC monolayer. Following incubation at $37^{\circ} \mathrm{C}$ for $24 \mathrm{~h}$, non-migrated cells were removed. Transinvaded cells were fixed and counted.

Statistical analysis. The statistical program SPSS 12.0 (SPSS, Chicago, IL, USA) was used to perform the statistical analyses. Statistically significant differences were determined using Student's t-test and $\mathrm{P}<0.05$ was considered to indicate a statistically significant difference.

\section{Results}

Poor prognosis for patients with muscle invasive bladder cancer. Patients receiving radical cystectomies $(n=324)$ were divided into two groups (non-muscle invasion, $n=104$ and muscle invasion, $n=204$ ) according to the pathological state of invasion. Fig. 1A and B show the representative images of non-muscle invasive bladder cancer and muscle invasive bladder cancer, respectively. Non-muscle invasive bladder cancer exhibited papillary growth (Fig. 1A), whereas numerous cancer cells were observed in the muscle layer in muscle invasive bladder cancer as indicated by the black arrows in Fig. 1B. Of the 324 patients, cancer recurrence was exhibited in 84 patients. The recurrence rate was higher in the muscle invasion group (87\%) compared with the non-muscle invasion group (13\%; Fig. 1C). Furthermore, the Kaplan-Meier curve demonstrated that overall survival was significantly shorter in the muscle invasion group compared with the non-muscle invasion group $(\mathrm{P}<0.0001$; Fig. 1D). These results indicate that the muscle invasiveness of bladder cancer correlates with a higher recurrence rate and a worse prognosis.

Invasion capacities of bladder cancer cells. To further characterize muscle invasive and non-muscle invasive bladder cancers, primary cultures of bladder cancers were established for various assays. Primary culture cells were prepared from bladder tumor specimens of four patients. Patients 1 and 3 (P1 and P3) had muscle invasive cancer and P2 and P4 had non-muscle invasive cancer (Fig. 2A). The cells were initially tested for their Matrigel invasion capacity. For comparison, two established bladder cancer cell lines, RT4 and YTS-1, were adopted. RT4 was derived from a well-differentiated papillary tumor and YTS-1 was established from an undifferentiated high-grade urothelial carcinoma, which is muscle 


\begin{tabular}{lccc}
\hline & Age & Gender & Muscle invasion \\
\hline Patient 1 & 45 & $\mathrm{~F}$ & + \\
Patient 2 & 75 & $\mathrm{~F}$ & - \\
Patient 3 & 76 & $\mathrm{M}$ & + \\
Patient 4 & 57 & $\mathrm{M}$ & - \\
\hline
\end{tabular}

B

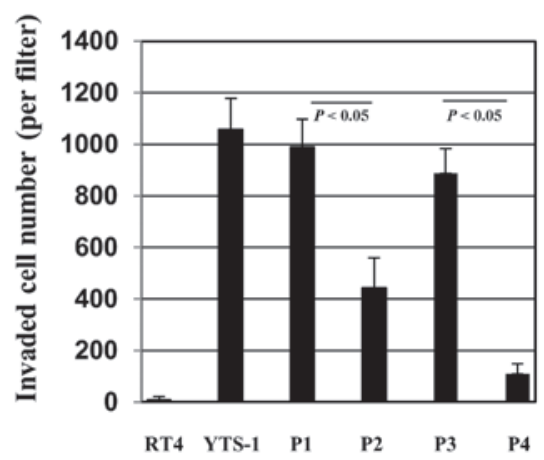

Figure 2. Matrigel invasion capacity of primary culture cells. (A) Profile of bladder cancer patients. Primary culture cells were prepared from four genetically independent patients. + , muscle invasive cancer; -, non-muscle invasive cancer. (B) Four primary culture cells, patient (P)1, P2, P3, P4, and two established bladder cancer cell lines, RT4 and YTS-1 were tested for Matrigel invasion in a Boyden chamber assay. Carboxyfluorescein diacetate succinimidyl ester-labeled cells that migrated through the Matrigel matrix were counted under a fluorescence microscope. Data are presented as means \pm standard deviation of triplicate measurements.

invasive. YTS-1 exhibited a high Matrigel invasion capacity, however, the invasion capacity of RT4 was particularly low (Fig. 2B). The primary culture cells from P1 and P3 with muscle invasive cancer exhibited high Matrigel invasion capacities, which are equivalent to that of YTS-1 (Fig. 2B). By contrast, the invasion capacities of the primary culture cells from P2 and P4 with non-muscle invasive cancer were significantly lower than those of P1 and P3 (Fig. 2B). These results indicate that muscle invasive bladder cancer cells exhibited a higher capacity for Matrigel invasion compared with non-invasive bladder cancer cells.

For bladder cancer cells to invade the muscle layer, they must invade through a urothelial cell monolayer. In order to evaluate the capacity of invasion through the urothelial cells, we established a transurothelial invasion system using HUCs (Fig. 3A). HUCs, the cells lining the surface of the bladder, are the first line of defense for the bladder against infection by various pathogens and cancer cell invasion. Bladder cancer cells were placed onto a monolayer of HUCs (Fig. 3B) and $24 \mathrm{~h}$ later the transurothelial invasion capacity of the bladder cancer cells was measured by counting the number of cells that had transinvaded through the HUC monolayer. YTS-1 exhibited a higher capacity for transurothelial invasion capacity and the invasion capacity of RT4 was particularly low (Fig. 3C). Primary culture cells from muscle invasive bladder cancer (P1 and P3) also exhibited a significantly higher capacity for transurothelial invasion compared with the primary culture cells from non-muscle invasive bladder cancer (P2 and P4) (Fig. 3C). These results indicate that muscle invasive bladder cancer cells possess a significantly
A

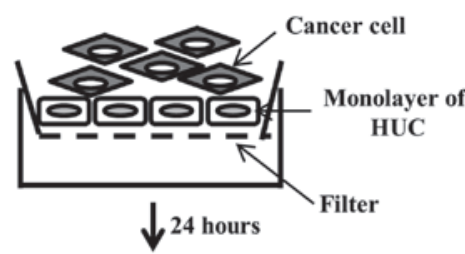

B
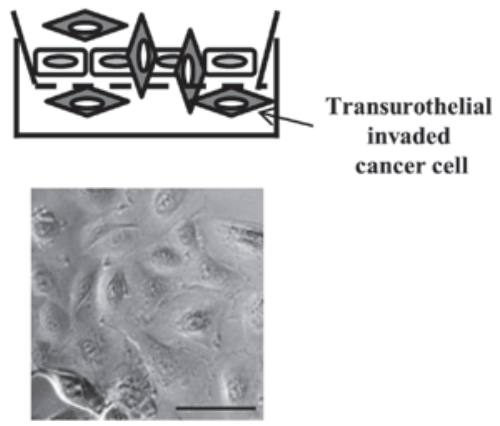

invaded
cancer cell

C

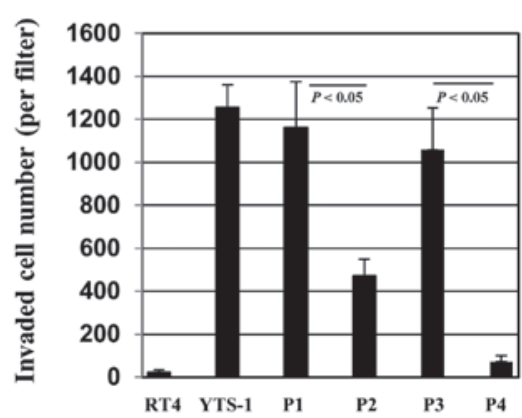

Figure 3. Transurothelial invasion capacity of primary culture cells (A) Schematic drawing of transurothelial invasion assay. (B) Monolayer of HUCs grown on the top surface of the filter of the cell culture insert. Scale bar, $50 \mu \mathrm{m}$. (C) Four primary culture cells; P1, P2, P3 and P4, and two bladder cancer cell lines, RT4 and YTS-1, were tested for transurothelial invasion. Carboxyfluorescein diacetate succinimidyl ester-labeled cells that invaded through the HUC monolayer were counted under a fluorescence microscope. Data are presented as means \pm standard deviation of triplicate measurements; HUC, human urothelial cell; $\mathrm{P}$, patient.

higher capacity for transurothelial invasion compared with non-muscle invasive cells.

Invadopodia formation in bladder cancer cells. Invasive cancer cells form invadopodia to invade the surrounding tissues; these filamentous actin (F-actin)-based membrane protrusions are required for the degradation of the extracellular matrix (ECM) and migration through the tissues (7-9). The primary culture cells were analyzed for the formation of invadopodia. Cells were double-stained with phalloidin and a cortactin monoclonal antibody (clone EP1922Y), which acts as an invadopodium marker (10-12). Invadopodia are visualized as yellow puncta, since the cores of invadopodia are composed of F-actin and cortactin co-localizes with F-actin. The typical invadopodia are denoted by white arrows in Fig. 4. Few invadopodia were observed in RT4 cells (Fig. 4A-C), however, numerous invadopodia were formed in the YTS-1 cells (Fig. 4D-F). Furthermore, primary culture cells from muscle invasive bladder cancer (P1 and $\mathrm{P} 3$ ) formed numerous invadopodia (Fig. 4G-I and M-O). By contrast, primary culture cells from non-muscle invasive bladder cancer 


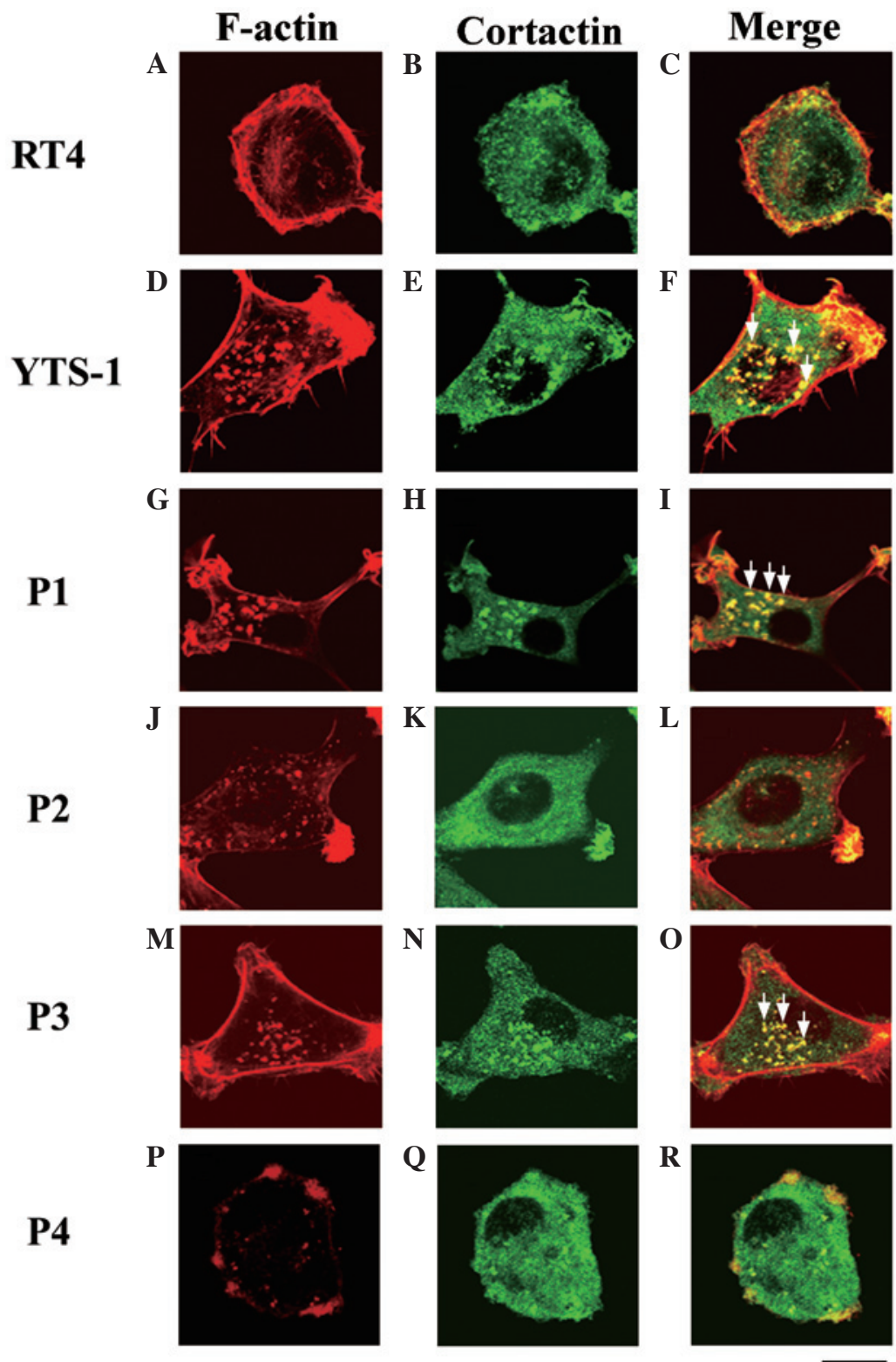

Figure 4. Invadopodia formation by bladder cancer cells. Two bladder cancer cell lines: (A-C) RT4, non-invasive cancer cells; (D-F) YTS-1, invasive cancer cells; and primary culture cells from four patients: (G-R) P1, P2, P3 and P4, were double-stained for invadopodia formation by phalloidin and anti-cortactin (an invadopodium marker). (A, D, G, J, M and P) F-actin staining (red), (B, E, H, K, N and Q) cortactin staining (green), and (C, F, I, L, O and R) a merge of F-actin and cortactin staining. Yellow indicates the co-localization of F-actin and cortactin. Certain typical invadopodia are exhibited in the YTS-1, P1 and P3 cells, and are denoted by white arrows. Scale bar, $20 \mu \mathrm{m}$. P, patient.

(P2 and P4) formed very few invadopodia (Fig. 4J-L and P-R). These results indicate that the muscle invasiveness of bladder cancer cells correlates with the ability to form invadopodia.

Secretion of metalloproteinases (MMPs) by bladder cancer cells. In order to degrade surrounding tissues and the ECM for invasion, invadopodia secrete MMPs, which is a primary function of invadopodia. It has previously been demonstrated that the ability of bladder cancer cells to form invadopodia correlated with the quantity of MMP-2 secretion (13). The secretion of MMP-2 by bladder cancer cells was subsequently examined by gelatin zymography. A large quantity of MMP-2 was secreted by YTS-1 and the muscle invasive bladder cancer cells from P1 and P3 (Fig. 5, lanes 3, 4 and 6). By contrast, the non-muscle invasive cancer cells, such as the RT4 cells

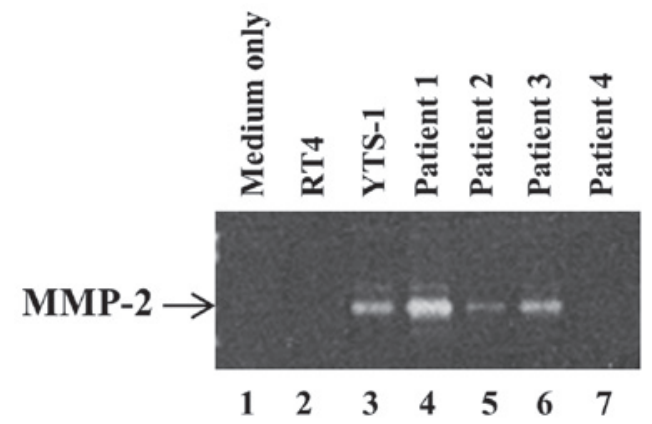

Figure 5. Secretion of MMP-2 by bladder cancer cells. Conditioned media from bladder cancer cells were collected and assayed for gelatinase activity by zymography. Representative zymograms from lane 1, medium only; lane 2, RT4; lane 3, YTS-1; lanes 4-7, patients 1-4. The MMP-2 bands appear as a clear band against dark background. MMP-2, metalloprotinase-2; RT4 and YTS-1, bladder cancer cell lines. 
A

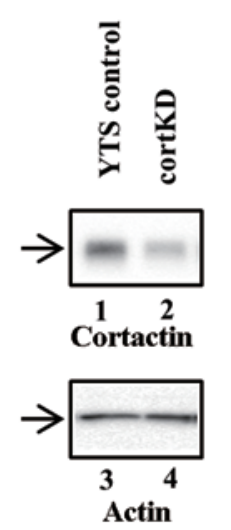

B

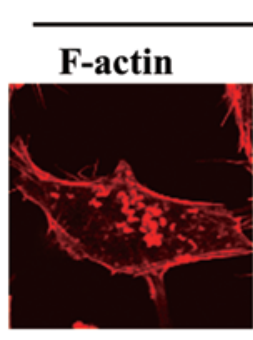

C

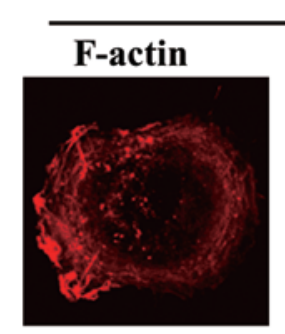

YTS control

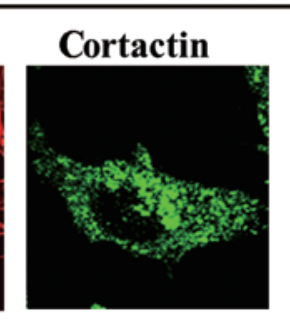

cortKD

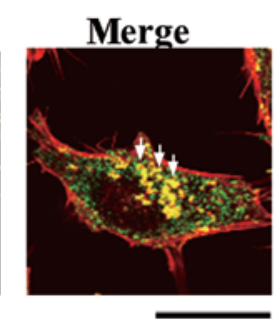

D
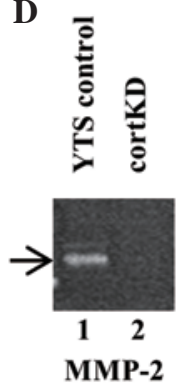

$\mathbf{E}$

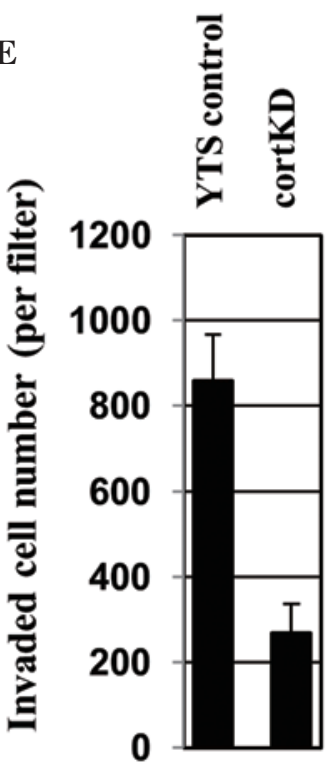

Figure 6. Invadopodia formation ability and transurothelial invasion capacity were reduced in cortKD. (A) Expression level of cortactin was reduced in cortKD. Total protein samples from the YTS control cells and corKD were analyzed by western blotting for the expression of cortactin and actin. (B) Invadopodia formation by the YTS control cells. (C) Invadopodia formation by cortKD. Cells were double-stained by phalloidin and anti-cortactin for invadopodia formation. (B and C) Confocal micrographs of (left panels) F-actin staining (red), (central panels) cortactin staining (green), and the (right panels) merge of F-actin and cortactin staining. Yellow indicates the co-localization of F-actin and cortactin. Certain typical invadopodia are observed in the YTS-1 control cells and denoted by white arrows. Scale bar, $20 \mu \mathrm{m}$. (D) Secretion of MMP-2 was reduced in cortKD. Conditioned media from the YTS control cells and cortKD were assayed for gelatinase activity by zymography. Representative zymograms from the YTS control cells (lane 1) and cortKD (lane 2). (E) Transurothelial invasion capacity of cortKD was reduced. The cells were assessed for transurothelial invasion through the human urothelial cell monolayer under a fluorescence microscope. Data are presented as means \pm standard deviation of triplicate measurements. YTS, bladder cancer cell line; cortKD, cortactin knockdown bladder cancer cells; MMP-2, metalloprotinase-2.

and those from $\mathrm{P} 2$ and $\mathrm{P} 4$ only secrete a marginal quantity of MMP-2, if any (Fig. 5, lanes 2, 5 and 7).

Effect of the reduction of invadopodia formation on transurothelial invasion. It was demonstrated that YTS-1 and muscle invasive bladder cancer cells from P1 and P3 exhibited a greater ability to invade through the urothelial cell monolayer (Fig. 3) and that these cells formed functional invadopodia (Figs. 4 and 5), indicating that invadopodia are important in bladder cancer cell invasion through the urothelial cell monolayer. In order to determine the importance of invadopodia in transurothelial invasion, the formation of invadopodia in YTS-1 cells was reduced and the effect of this reduction on transurothelial invasion was examined. To lower the formation of invadopodia, the expression of cortactin in YTS-1 cells was silenced, as cortactin is a key regulator for invadopodia formation (10-12).

Several stable cortactin knockdown cell lines were established using an invasive bladder cancer cell line, YTS-1. A YTS-1 cell line expressing non-targeting siRNA served as a control (designated YTS control). Western blotting revealed that cortactin expression was lower in a knockdown cell line (designated cortKD) compared with the YTS control (Fig. 6A). The results from the assays using cortKD are shown in the present study, since the results yielded from other cortactin knockdown cell lines were almost identical to those from cortKD in all of the assays. Invadopodia in the YTS control cells were visualized using Alexa Fluor ${ }^{\circledR}$ 568-labeled phalloidin staining as F-actin-rich puncta (Fig. 6B, left panel). A section of cortactin staining also exhibited a punctate pattern (Fig. 6B, central panel) and co-localization of cortactin with F-actin puncta, which indicates that these puncta are invadopodia (Fig. 6B, right panel). Certain typical invadopodia are indicated by the white arrows (Fig. 6B, right panel). In cortKD cells, no clear invadopodia were observed (Fig. 6C). These results indicate that the formation of invadopodia was impaired in cortKD cells. The cells were subsequently assayed for the secretion of MMPs. According to gelatin zymography, a large quantity of MMP was secreted by the YTS control cells (Fig. 6D, lane 1), however, the secretion of MMP-2 by cortKD cells was significantly lower than that secreted by the YTS control cells (Fig. 6D, lane 2).

cortKD cells impaired the formation of invadopodia and the secretion of MMPs (Fig. 6A-D). To examine whether impairing invadopodia formation affects transurothelial invasion, the transurothelial invasion capacity of cortKD and YTS control cells was measured. The transurothelial invasion capacity of cortKD cells was markedly reduced compared with that of the YTS control cells (Fig. 6E). This indicates that impaired invadopodia formation and reduced MMP-2 secretion result in reduced transurothelial invasion, which indicates that invadopodia are essential in the invasion of bladder cancer cells through the HUC monolayer.

\section{Discussion}

Muscle invasive bladder cancer requires radical treatments, including cystectomy with pelvic lymph node dissection. 
Although muscle invasion is a crucial process for metastasis and prognosis, little is known concerning its cellular and molecular mechanisms. In the present study, a novel method was developed to assay the invasion through the urothelial cell monolayer using HUCs (Fig. 3A). Transurothelial invasion of bladder cancer cells is a crucial prerequisite for muscle invasion. To the best of our knowledge, this is the first study to assay and compare the ability to invade through the urothelial cell monolayer between bladder cancer cell types.

In order for bladder cancer cells to invade the muscle layer, the cells are required to invade through the urothelial cell monolayer and the basement membrane (14). Transurothelial invasion mimics the invasion of bladder cancer cells through the urothelial cell monolayer. Matrigel invasion represents the degradation of basement membrane by cancer cells, due to the fact that Matrigel matrix gel consists of the components of the basement membrane (15). Therefore, the ability of the bladder cancer cells to invade the muscle layer may be evaluated by measuring transurothelial invasion and Matrigel invasion capacities. The results of the present study (Figs. 2 and 3) demonstrate that the Matrigel and transurothelial invasion capacities of cancer cells derived from invasion bladder cancer (YTS-1, P1 and P3) were markedly higher than those of cells derived from non-muscle invasive bladder cancer (RT4, P2 and P4).

Invadopodia were originally identified and have been investigated as membrane protrusions that are required for the degradation of the ECM and migration through the tissues (7-9). It has been reported that invadopodia are essential in the intravasation of cancer cells from the primary site into the blood vessels (16). However, the importance of invadopodia formation in other processes during hematogenous metastasis is not understood. The present study revealed that invadopodia formation is involved in the transurothelial invasion process. To date, numerous attempts have been made to understand the molecular mechanisms underlying the formation of invadopodia. Further investigation into the molecular processes of invadopodia formation may lead to the development of 'anti-muscle invasion' agents or therapeutic methods for the treatment of bladder cancer.

\section{Acknowledgements}

This study was supported by grants-in-aid for Scientific Research from the Japanese Society for the Promotion of Science (grant no. 22570131, to S.T. and grant no. B22390301, to C.O.), the Ministry of Education, Culture, Sports, Science and Technology of Japan (grant no. 21791483, to C.O. and grant no. 21791484, to T.K.) and Japan Science and Technology Agency (CREST; to C.O.).

\section{References}

1. Steeg PS: Tumor metastasis: mechanistic insights and clinical challenges. Nat Med 12: 895-904, 2006.

2. Tsuboi S, Sutoh M, Hatakeyama S, et al: A novel strategy for evasion of NK cell immunity by tumours expressing core 2 O-glycans. EMBO J 30: 3173-3185, 2011.

3. Suzuki Y, Sutoh M, Hatakeyama S, et al: MUC1 carrying core 2 $\mathrm{O}$-glycans functions as a molecular shield against NK cell attack, promoting bladder tumor metastasis. Int J Oncol 40: 1831-1838, 2012.

4. Tsuboi S, Hatakeyama S, Ohyama C and Fukuda M: Two opposing roles of O-glycans in tumor metastasis. Trends Mol Med 18: 224-232, 2012.

5. Vishnu P, Mathew J and Tan WW: Current therapeutic strategies for invasive and metastatic bladder cancer. Onco Targets Ther 4: 97-113, 2011.

6. Eble JN, Sauter G, Epstein JI, Sesterhenn IA (Eds.): World Health Organization Classification of Tumours. Pathology and Genetics of Tumours of the Urinary System and Male Genital Organs. IARC Press, Lyon, France, 2004.

7. Caldieri G, Ayala I, Attanasio F and Buccione R: Cell and molecular biology of invadopodia. Int Rev Cell Mol Biol 275: $1-34,2009$.

8. Linder S, Wiesner C and Himmel M: Degrading devices: invadosomes in proteolytic cell invasion. Annu Rev Cell Dev Biol 27: 185-211, 2011.

9. Murphy DA and Courtneidge SA: The 'ins' and 'outs' of podosomes and invadopodia: characteristics, formation and function. Nat Rev Mol Cell Biol 12: 413-426, 2011.

10. Artym VV, Zhang Y, Seillier-Moiseiwitsch F, Yamada KM and Mueller SC: Dynamic interactions of cortactin and membrane type 1 matrix metalloproteinase at invadopodia: defining the stages of invadopodia formation and function. Cancer Res 66: 3034-3043, 2006.

11. Ayala I, Baldassarre M, Giacchetti G, et al: Multiple regulatory inputs converge on cortactin to control invadopodia biogenesis and extracellular matrix degradation. J Cell Sci 121: 369-378, 2008.

12. Oser M, Yamaguchi H, Mader CC, et al: Cortactin regulates cofilin and N-WASp activities to control the stages of invadopodium assembly and maturation. J Cell Biol 186: 571-587, 2009.

13. Sutoh M, Hashimoto $\mathrm{Y}$, Yoneyama T, et al: Invadopodia formation by bladder tumor cells. Oncol Res 19: 85-92, 2010.

14. Jewett HJ and Strong GH: Infiltrating carcinoma of the bladder; relation of depth of penetration of the bladder wall to incidence of local extension and metastases. J Urol 55: 366-372, 1946.

15. Kleinman HK and Jacob K: Invasion assays. Curr Protoc Cell Biol Chapter 12: Unit 12.2, 2001.

16. Gligorijevic B, Wyckoff J, Yamaguchi H, Wang Y, Roussos ET and Condeelis J: N-WASP-mediated invadopodium formation is involved in intravasation and lung metastasis of mammary tumors. J Cell Sci 125: 724-734, 2012. 